\title{
Investigación social
}

sobre las artes marciales

\section{extremo-orientales en}

\section{Polonia}

\author{
WOJCIECH J. CYNARSKI \\ Universidad de Rzeszów (Polonia)
}

Recibido 27/09/2009 - Aceptado 16/11/2009

\section{Resumen}

日 presente estudio resume los logros de los investigadores polacos sobre el fenómeno sociocultural de las artes marciales, observado desde la pespectiva de la historia centenaria de estas prácticas en Polonia y la larga tradición de formes marciales locales, así como desde la experiencia de los más de treinta años de autor como practicante y posteriormente investigador de las artes marciales. Seconsideran las principales direcciones de investigación en el ámbito multidisciplinar, sociológico, histórico y pedagógico, e igualmente se describe la institucionalizacón de las actividades científicas ligadas a las artes marciales, tales como la organización de conferencias periódicas y la publicación de revistas específicas. Particularmente, se detallan los logros alcanzados en el centro de investigación de Rzeszów, que se está convirtiendo en un centro internacional de referencia en el estudio científico de las artes merciales.

Palabras dave artes marciales, investigación científica, teóricos, direcciones fundamentales de investigación.

\section{Social research of Far-Eastem martial arts in Poland}

Abstract: The present study summerizes the achievements of the polish researchers about the socio-cultural phenomenon of martial arts, shown from the historical perspective of these practices in Poland and the long-time tradition of local martial practices, as well as from the author's thirty-year of practice and research in martial arts fied. The main directions of the multidisciplinary, sociologica, historical and pedagogical research are considered, in addition to the institutionalization of scientific activities related to the martial arts such as the organization of periodic conferences and the publication of specific journals. Particularly, the adhievements of the Rzeszów's Research Center, which is becoming an intemational comerstone in the scientific study of the martial arts, are detailed.

Key words: martial arts, scientific research, theoreticians, main directions of research.

\section{Pesquisa Social solbre as Artes Marciais na Polónia}

Resumo: O presenteestudo resume os resultados alcançados pelos investigadores polacos sobre o fenómeno sociocultura das artes marciais, observado desdea perspectiva da história centenária destas práticas na Polónia e a larga tradição de formas merciais locais, assim como desde a experiência dos mais de trinta anos do autor como praticante e, posteriormente, investigador das artes marciais. Consideramse como principais linhas de investigação: a multi-disciplinar, a sociológica, a histónica e a pedagógica Descrevemos, igualmente, a institucionalização das actividades científicas ligadas às artes marciais, tais como a organização deconferências periódicas 
e a publicação de revistas específicas e se detalham os resultados alcançados no centro de investigação de Rzeszów que se está convertendo num centro internacional de referência de estudo científico sobre as artes marciais.

Palavras-chave: artes marciais, investigação científica, teóricos, direcções fundamentais de investigação.

PORTADA DE UBRO MARTIAL ARTS \& COMBAT

SPORTS - HUMANISTIC OTLOOK [ARTES MARCIALE Y DEPORTES DE COMBATE - UNA PERSECTIVA HUMANISTA], EITADO POR LA UNIVESIDAD DE RZESZÓW Y COORDINADO POR E

CYNARSKI. EN ESTA OBRA SE REÚNEN ALGUNOS DE LOS TRABAJOS PRESENTADOS 日N LA “2ND INTERATIONAL

Salentiac Confrance Martial Arts, Combat SPORTS AND HUMANISM - BUDŌ, KAKUGI, JINDō", CEMRADA EN LAS CIUDADES DE TARGOWISKA Y KROSNO ENTRE В 25 Y 26 DE ABRIL DE 2008.

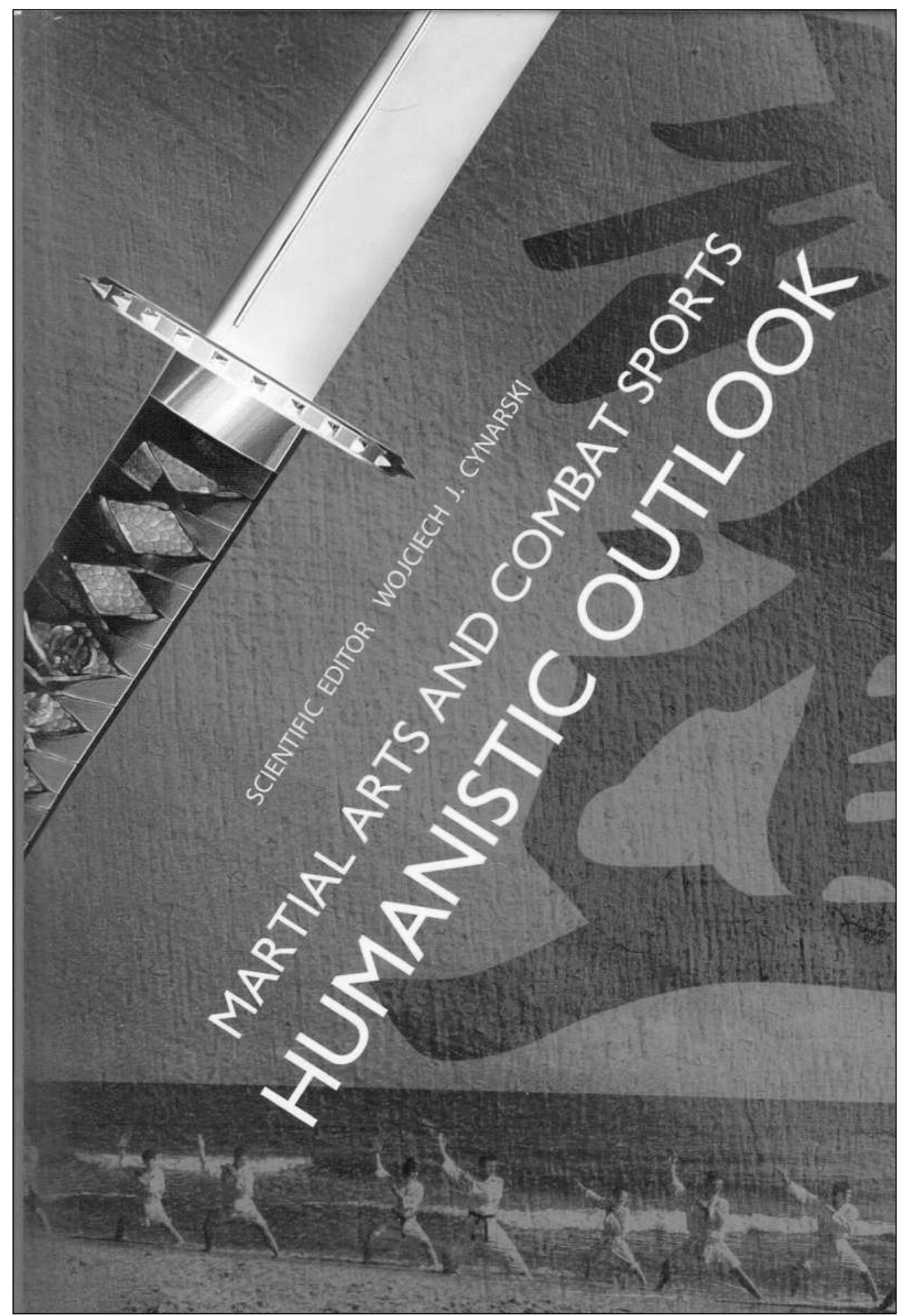




\section{Investigación social}

sobre las artes marciales

\section{extremo-orientales en}

\section{Polonia}

WOJCIECH J. CYNARSKI

Universidad de Rzeszów (Polonia)

\section{Introckucción}

La sociología de deporte, junto con la sociología de la actividad física y la sociología de la recreación, constituyen la sociología de la cultura física Esta sociología define la mirada socio-cultural hacia las artes marciales, creando una sociología de las artes marciales que está influenciada por los resultados de la investigación y las tesis derivadas de otras sub-disciplinas sociológicas y áreas relacionadas (antropología cultural, sociología cultural, sociología del espacio, sociología organizacional, filosofía social, psicología social, etc.). Sobre la base de una relativamente reciente, pero importante tradición de estudios en el ámbito de las artes marciales, este trabajo presenta los notables avances de los teóricos e investigadores polacos que participan enégicamente en la sociedad sociológica europea y en el grupo intemacional asociado con la revista ldō - Ruch dla Kultury / Movement for Culture (IRK-MC). Igualmente, también se presentan los éxitos organizacionales e institucionales más importantes en el estudio académico de las artes marciales en Polonia, que desde hace algunos años se ha convertido en un importante lugar de encuentro y debate para investigadores a nivel intemacional.

\section{Direcciones y resultados de la investigación en el ámbito socio-cultural de las artes marciales en Polonia}

日 primer experto polaco en deportes de combate y artes marciales que abordó la explicación científica de estas actividades desde una perspectiva humanística y sociocultural fue Stanistaw Tokarski. Como filósofo, orientalista (indologista y estudiante de Mircea 日iade) y entrenador de judo (4 dan), explicó el significado de las artes marciales en consonancia con las ideas de Jigoro Kano. Tokarski identifica las artes marciales y los deportes de combate asiáticos señalando como elementos comunes la auto-expresión, la auto-realización y la defensa personal, definiendo las artes merciales como "formas de actividad de la expresión de la filosofía oriental" (Tokarski, 1989). Si bien no es una definición precisa, lo que trata el autor es de llamer la atención sobre el carácter expre sivo de estas prácticas. Así, Tokarski interpreta las artes marciales en el amplio contexto teórico de la nueva antropología humenista de la Escuela de Chicago (Mircea Eiade) y de las teorías del diálogo cultural (Tokarski, 1976). Tokarski desamolla su investigación en e Centro de Estudios de Paises No-Europeos, en la Academia Polaca de Ciencias, y la mayoría de sus trabajos pueden ubicarse en el ámbito de los estudios culturales. 
Una tendencia de investigación similar, aunque desde una perspectiva inter y multi-disciplinar més amplia, es la realizada por Wojciech J. Cynarski, cuyas dos obras dedicadas a los diálogos culturales (Cynarski, 2000, 2008a) tuvieron a Tokarski como revisor editorial. Este autor representa simultáneamente a las ciencias de deporte y a la sociología, e igualmente es un veterano practicante de artes merciales. Junto con otros científicos y expertos asociados a la Idokan Poland Association (IPA) y a la revista IRK-MC, ha creado una Teońa Humanística de las Artes Marciales (Cynarski \& Obodyński, 2003b; Cynarski, 2004). También ha formulado una filosofía de las artes marciales no-históricas practicadas actualmente a escala global (Kosiewicz, 2007). Cynarski fundamenta todo su estudio de las artes marciales en una nueva aproximación metodológica, paradigmática y sistemática, y en una nueva antropología como teońa sintética del hombre, la cultura y la sociedad (Cynarski, 2004). Como define con precisión el profesor de filosofía Jerzy Kosievicz, "La tendencia hacia una aproximación sistemética e interdisciplinar puede ser el resultado de la formación multi-direccional de Cynarski en ingeniería, pedagogía, entrenamiento y sociología. $日$ autor utiliza un método de observación participativa, haciendo uso de su experiencia directa como competidor, entrenador, activista y árbitro, así como una persona que estudia los estilos y escuelas dásicas de artes marciales en Europa y en Japón" (Kosievic, 2005).

Los elementos de pensamiento humenístico, principalmente desde la perspectiva pedagógica, fueron induidos en los trabajos de Ewaryst Jaskólski (1932-2007), profesor de ciencias de la cultura física y además un excelente entrenador de judo (50 dan). Su trabajo describe los valores educativos resultantes de la práctica del judo y otras artes marciales. Jaskólski (2000) ha llamado la atención sobre cómo mediante las artes marciales se produce un desarrollo combinado de la personalidad y de la "disposición" de cuerpo. Además, junto con J. Migasiewicz y K. Kurzawski, E Jaskólski ha creado una pedagogía de las artes merciales basada en la pedagogía de la educación física, pero que también se relaciona con la pedagogía tradicional de las artes merciales y las aportaciones teónicas de otros expertos polacos (Jaskólski, Migasiewic \& Kurzawski, 2008).

Otro de los expertos polacos es el profesor Romen Maciej Kalina, estudiante de Jaskólski y representante de las ciencias de la cultura física Kalina ha desarollado las ideess del profesor Jaskólski, creando una Teoría de los Deportes de Combate (Kalina, 2000). Aquí, la perspectiva teórica constituye principalmente una teoría general praxiológica de combate Kalina enfatiza los valores educativos y utilitarios que tienen que ver tanto con la defensa personal como con las artes marciales y los deportes de combate Otra de sus importantes aportaciones es la creación de la revista electrónica Archives of Budo (http://wnww.archbudo.com/), la cual publica fundamentalmente trabajos de investigación desde la perspectiva biotécnica de las ciencias del deporte, así como contenidos humanísticos.

Desde la perspectiva pedagógica, la contribución més importante a la explicación de las técnicas de las artes marciales se atribuye a Andrzej Szyszko-Bohusz, fundador de la Pedagogía Holística Como pedagogo, experto en religión, psicólogo, filósofo y experto en las tradiciones místicas y en kárate es, de alguna manera, un co-fundador de la Teoría Humenística de las Artes Marciales (compárese con Szyszko-Bohusz, 2003, 2008a). En sus enseñanzas Szyszko-Bohusz enfatiza las consideraciones de filósofo indio Vivekanandy (nombre propio Narendranath Dutta, 1863-1902) y de Papa Juan Pablo II (1920-2005), aconsejando que las personas han de ponerse en contacto con la realidad a través de la introspección. Su teoría está centrada explícitamente en Jesucristo, ya que considera que é es el único gunú, maestro y profesor. 日 profesor Szyszko-Bohusz cita las funciones pedagógicas de las artes marciales relacionadas con la educación, tan- 
to aquellas propiamente educativas como las complementarias, enumerando su acción sobre componentes de la personalidad tales como el cuenpo, la energía y la inteligencia En este sentido, los comportamientos requeridos a los estudiantes del budo son concentración, auto-control y confianza (Szyszko-Bohusz, 2008b). En la figura de Andrzej Szyszko-Bohusz podemos observar la combinación de la ética humanística cristiana con la aproximación, libre de prejuicios, hacia el conocimiento oriental.

Por otra parte, el profesor Andrzej Pantudd, representando e ámbito humanístico de las ciencias del deporte y la pedagogía del deporteen Polonia, ha presentado algunas ideas inspiradoras a través de su crítica de las artes marciales como fenómeno sociocultural. Refiniéndose a los trabajos de Cynarski, Roland J. Maroteaux y otros autores (Cynarski \& Obodyński, 2003b; Maroteaux \& Cynarski, 2002-2003), Pantudk escribe desde una perspectiva crítica sobre la "asiatización" de la cultura deportiva europea (Pantudk, 2004; 2007), temas que los citados autores habían tratado de forma diferente (Cynarski, 2008c; Tokarski \& Sikorski, 2009). Para los estudiantes de artes marciales, resultará particulamente interesante el capítulo del libro de Panłudk titulado "Asian body cult in the Polish way -imperialism or diffusion" [" $\mathrm{\theta}$ culto asiático del cuenpo a la manera polaca -imperialismo o difusión"], en el que polemiza con los estudiosos asociados a la revista IRK-MC. Esta discusión científica constructiva tendrá con toda seguridad una influencia sobre el desarrollo de la Teoría Humanística de las Artes Marciales en su aspecto pedagógico.

Por su parte la investigación histórica sobre las artes marciales todavia no ha avanzado mucho. Sólo recientemente ha aparecido un libro elaborado de acuerdo a la metodología de la investigación histónica, publicado en polaco e inglés. Aunque contiene algunas deficiencias, errores e imprecisiones, aún es indudablemente uno de los mejores estudios respecto a la historia de jujutsu en Polonia Los autores de esta monografía son dos investigadores de la ciudad de Glogów, Romen Grzegorz y Marcin Walendowicz (2008), ambos practicantes de jujutsu. Además, también se han realizado algunas contribuciones a la historia de las artes marciales en publicaciones secundarias (Cynarski, 1997; 2005c). Aquí encontramos trabajos relacionados con la historia del judo (Błach et al., 2006) y otras artes marciales asiáticas (Sieber \& Cynarski 2004; Cynarski \& al., 2006). También es interesante destacar las referencias a la tradición de más de mil años del ejército y de las artes merciales históricas polacas, llevada a cabo principalmente por investigadores relacionados con la esgrima (Zabłodki, 2000; Borysiuk, 2005; Czajkowski, 2007; Cynarski, 2008b).

\section{Sociología de las artes manciales en Poloniax problemas y perspectivas}

日 interés sociológico por e fenómeno de las artes merciales asiáticas se ha abordado desde la perspectiva de la sociología del deporte y desde la perspectiva más general de la teoría de los diálogos culturales. La primera de estas áreas está aún poco desamollada en Polonia (Cynarski \& Obodyński, 2004b), lo que puede deberse al escaso conocimiento de los sociólogos del deporte polacos de esta área de la cultura física que constituyen las artes merciales practicadas hoy en día a nivel mundial. La segunda perspectiva, centrada en la cultura y en los sistemes, parece despertar un meyor interés. Esta área de los diálogos culturales se refiere a diferentes formas de cultura, lo que nos permite la interpretación de las artes merciales en sus enormemente variadas formas psico-físicas.

Una revisión sobre los avances actuales relacionados con el estudio social de las artes merciales es la que se presenta en el trabajo "Sociology of Far Eastem martial arts problems and perspectives" ["Sociología de las Artes Marciales extremo-orientales - pro- 
blemes y perspectivas"], publicado en Archives of Budo (Cynarski \& Obodyński, 2007). Este trabajo caracterizó independientemente las bases teóricas de esta sub-disciplina sociológica (Cynarski \& Obodyński, 2003a; Cynarski, 2004, 67-329).

Una de las direcciones interesantes de la investigación sociológica es la descripción de la percepción y evolución de las artes marciales, su occidentalización y modificación. La comiente de investigación de los autores europeos (Hartl, Faber \& Bögle, 1989; Echberg, 2004; Villamón \& al. 2004), americanos (Donohue, 1994, 1997; Orlando, 1997) y asiáticos (Chiba, Ebihara \& Morino, 2001) corre pardela a la de algunos de los autores polacos (Tokarski, 1989; Cynarski \& Obodyński, 2005; Cynarski, Sieber \& Litwiniuk, 2005, 2006). Uno de los elementos de reflexión recurrentes es si, en estos tiempos de globalización, jpresenvarán las artes marciales su identidad, o se convertirán en materias de intercambio en el mercado global, a modo de deporte contemporáneo (Stekkowicz, 2001; Obodyński \& Cynarski, 2003)? Otro tema tratado habitualmente es la institucionalización de las artes marciales (Cynarski \& Obodyński, 2005; Cynarski 2006b) y e estudio del desarollo organizativo de los clubes, asociaciones y federaciones de varias artes marciales (Cynarski 2003; Sieber \& Cynarski, 2004; Cynarski, Obodyński, Litwiniuk \& Cynarska, 2006).

La Teoría de los Diálogos Culturales aplicada a las artes marciales ofrece valiosos análisis comparativos e interpretaciones precisas sobre elementos trascendentales para su comprensión, como son los mensajes inter-culturales, las tradiciones, las percepciones, la adaptación a las condiciones y mentalidades locales, la creación de falsificaciones, etc. (Tokarski 1989; Cynarski, 2000; 2004; 2008a). Asimismo, muestra los problemes de las direcciones de influencia cultural, orientalización-occidentalización, funcionamiento de las artes marciales en el proceso de globalización cultural, presencia en los medios de comunicación, o los vínculos con las tradiciones locales y la identidad nacional (Cynarski, 1999, 2006a; Tokarski, 2003; Obodyński \& Cynarski, 2003).

日 cambio social de los roles de la mujer, la liberación femenina (también en la cultura de deporte) y la idea del feminismo encuentran eco en algunos discursos rea lizados en esta área de la sociología denominada "FMA" [i.e Far Easter Martial Arts Artes Marciales del Lejano Oriente, en polaco "DSW"]. Las mujeres sociólogas no sólo escriben solbre luchadoras femeninas contemporáneas (Förster, 1983; Castelnuovo \& Guthrie, 1998; Cox \& Thompson, 2000; Hovden, 2000; Maeda, 2000; McDonald, 2000; Mennesson, 2000; Socha, 2001; Speer, 2001), sino que también participan activamente y de forma considerable en las artes marciales. "Las amazonas están en amonía con e modelo de mujer post-modema y dinánica" (Cynarski, 2004, 289-307); las mujeres se liberan de algunos de sus roles tradicionales y adoptan otros asociados a las tradiciones de las artes marciales típicamente asociados a los hombres, compitiendo en disciplinas de combate como por ejemplo el boxeo.

Desde la perspectiva de la sociología del espacio, se ha analizado el espacio de práctica de las artes marciales (dojo en japonés - el lugar del camino) en su relación con dicho camino o trayectoria vital que sigue d practicante Los estudiosos explican e simbolismo de este lugar y su conexión con la antropología (filosófica y cultura) de las artes marciales (Cynarski, 2005a). La sala de ejercicios para la práctica del budo no es únicamente un lugar donde se entrena duro, sino que es también un lugar de desarrollo personal a través de esfuerzo constante de auto-creación, en el que la relación maestroprofesor resulta de gran ayuda. Las relaciones intenpersonales que tienen lugar en las escuelas de artes marciales han sido estudiadas por Sterkowic (2003) y Cynarski (2004).

Por otra parte, se han realizado intentos para describir e modo en que los repre sentantes de diferentes artes marciales y deportes de combate cumplen con el canon 
ético de fair play. Los estudios en ética descriptiva (Litwiniuk \& Cynarski, 2001) confirman la integridad de la ética nomativa, relacionada con esta idea de fair play, y la conducta (vital, mord) de los practicantes de artes marciales. Los resultados que arrojan los métodos de investigación cualitativos parecen ser muy prometedores (Ślęzak, 2006) en este ámbito de conocimiento.

Por último, el análisis de las relaciones entre las artes marciales, el marketing, la gestión y los negocios parece arrojar interesantes resultados (Pater, 1990/1999). Hasta e momento, esta línea de investigación ha sido asumida por especialistas americanos y japoneses, pero en Polonia no se ha desarrollado suficientemente aunque se ha iniciado la investigación en esta dirección (Mrówka, 2008).

La oportunidad para el progreso de la investigación social en las artes marciales es e tema 13 “Sociología de las artes marciales del Lejano Oriente - Investigación comparativa europea", dentro del proyecto Investigación interdisciplinar, multi-faceta sobre e fenómeno de las artes marciales (dirigido por W.J. Cynarski) realizado por el Comité de Investigación Científica de la IPA.

\section{Institucionalización de la investigación científica}

Desde d año 2000 se publica en Rzeszów la ya citada revista IRK-MC, fundada por los profesores Cynarski y Obodyński y dirigida actualmente por el primero de ellos. Reuniendo a un amplio pane de científicos polacos y de otros países, IRK-MC tiene como uno de sus principales objetivos el desarollar la Teoría Humanística de las Artes Marciales. IRK-MC se publica en papel con el apoyo del Comité de Investigación Científica de la IPA y de los miembros intemacionales de Comité Editorial y del Comité Científico. IRK-MC difunde artículos y estudios relativos a la historia de las artes marciales, estructuras organizativas, perfiles de maestros-expertos, trabajos sobre los procesos y los temas socio-culturales, afectando a los creadores de budo contemporáneo y a otras personas que crean la historia contemporánea de las artes marciales. La revista describe la historia de asociaciones y federaciones y la fundación y desarrollo de nuevas escuelas y estilos, incluyendo análisis de materiales de referencia e interpretaciones de hechos poco conocidos. También se induyen opiniones sobre temas específicos relacionados con las artes marciales y su particular cultura física, simbólica y espiritual. Desde una perspectiva més generd, también se publican interesantes textos relacionados con la teoría de los deportes, entrenamiento, investigación empíica así como revisiones de libros e informes sobre acontecimientos tanto del mundo científico como de las artes marciales.

En e año 2005, e profesor Kalina fundó la ya también citada Archives of Budo (AB) en Varsovia $A B$ es una revista anual editada en formato electrónico, y esta dirigida por el propio profesor Kalina, que trabaja en la Facultad de Educación Física de la Universidad de Rzeszów. La revista publica, en particular, textos relacionados con los temes de los deportes de combate, defensa personal, educación militar, deportes extremos y artes marciales.

Asimismo, en Polonia han tenido lugar varias conferencias científicas intemacionales dedicadas a las artes merciales. La primera de ellas fue organizada en Plock de 18 a 19 de enero de 2002 por R.M. Kalina Los participantes de Polonia, Japón y Ucrania presentaron 8 informes y mostraron pósteres con los resultados de sus investigaciones. De 30 de mayo al 1 de junio de 2003, en Rzeszów, se organizó la “ $1^{\text {\} } \text { International }$ Scientific Conference" sobre Teoría humanistica de las artes marciales y deportes de combate-conceptos y problemas, promovida por los profesores Obodyński y Cynarski. 日 carácter intemacional de la conferencia fue confirmado con la presencia de los miembros de Comité Científico y la inscripción y participación activa de 46 participantes de 9 
países (Bielomusia, Croacia, Francia, Japón, Alemenia, Polonia, Eslovaquia y Ucrania). 日 acontecimiento fue apoyado por IRK-MC y tuvo como resultado una monografía colectiva (Cynarski \& Obodyński, 2003b) en la que se exponen los resultados de los análisis teóricos, reflexiones filosóficas, estudios interdisciplinarios e investigación empírica pre sentados en la conferencia, que sumeron un total de 25 trabajos.

Rzeszów también fue e emplazamiento de " 1 "International Scientific Congress of Martial Arts and Combat Sports", entre e 22 y e 24 de septiembre de 2006. Sus organizadores fueron los profesores Kalina, Obodyński y Cynarski. Aparte de las reuniones científicas (se presentaron 71 trabajos), hubo una Gala de Budo con exhibiciones de maestros de artes marciales chinas y japonesas. La presentación principal fue realizada por Stanistaw Tokarski. Más de 200 personas participaron activamente en la conferencia, representando a 18 páses entre participantes y miembros del Comité Científico. Las actas de evento se publicaron en inglés, conteniendo resúmenes extendidos de los trabajos, entre los cuales figuraban aportaciones derivadas de investigaciones en el ámbito socio-cultural y humanístico.

Entre los días 25 y 26 de abril de 2008, las ciudades de Targowiska y Krosno fue ron el lugar de encuentro para la celebración de la "2nd International Scientific Conference" de investigadores expertos en artes marciales y humanistas, titulada "Martial Arts, Combat Sports and Humanism-Budō, Kakugi, Jind̄̄" y patrocinada por IRK-MC y AB. La conferencia estuvo apoyada por tres sociedades científicas. $日$ resultado de la conferencia es una monografía que contiene únicamente algunos trabajos seleccionados según su temática (Cynarski, 2009), por ejemplo, aquellos realizados por A.A. Figueiredo, C. Gutiérez, T. Sasaki, A. Szyszko-Bohusz. Otros trabajos presentados en esta conferencia ya han sido publicados en IRK-MC o AB.

\section{La Universidad de Rzeszóv y la escuela científica}

日 impulsor de la mayoría de las iniciativas de la Escuela Científica de Rzeszów es e profesor Kazimierz Obodyński, actualmente decano de la Facultad de Educación Física de la Universidad de Rzeszóv. Esta Facultad es co-fundadora del centro de investigación de artes marciales de Rzeszów, en el que funcionó entre 2006 y 2008 la única cátedra de deportes de combate existente en Polonia, hoy en día de nuevo activa En el pasado y ahora, el Departamento de Deportes de Combate ha estado trabajando en cooperación con la Cátedra de Ciencias Humanísticas, la Idokan Poland Association y otras socieda des científicas.

Como señaló el fundador de la escuela polaca de sociología del deporte, Zbigniew Krawczyk, entre las pocas escuelas que están trabajando en este momento en Polonia en el campo de la sociología, la Escuela de Rzeszów está obteniendo buenos resultados. La sociología de las artes marciales es uno de los principales puntos de interés en este centro de investigación. Merece la pena volver a mencionar que un numeroso círculo de estudiosos polacos y extranjeros están reunidos alrededor de la revista IRK-MC, íntimamente ligada a la Escuela de Rzeszów.

Aparte de la investigación y publicaciones realizadas por los representantes de la Escuela de Rzeszów, relacionadas con las líneas de investigación descritas más amiba, podemos encontrar algunos conceptos nuevos y estudios originales. Además de los estudios de investigación y teóricos (Cynarski, 1999, 2004, 2005b; Cynarski \& Obodyński, 2003a), los investigadores de Rzeszów han llevado a cabo proyectos con una gran im portancia de aplicación práctica. Se han daborado, sobre la base de investigaciones en e ámbito recreativo concemientes a actividades en escuelas, clubes y secciones de artes marciales, algunos programas educativos de artes marciales para la educación física o el 
deporte También se ha visto que un practicante o "guerrero" actual de las artes marcia les reconoce un ethos similar al canon de regulación del samurái y al ethos del caballero europeo, aunque en una versión más humanística Así, podemos hablar de la influencia y del valor de las artes marciales en las vidas de los instructores y los estudiantes de artes marciales (Obodyński \& Cynarski, 2005). También se han realizado investigaciones sobre metodología orientadas al desarrollo de la sociología y la teoría general sistemética de las artes marciales.

Otro tema original son las "formas tuństicas" del entomo de las artes marciales, en otras palabras ed "turismo de las artes merciales", que se ha estudiado desde la perspectiva de la antropología de las artes merciales, la teoría humanística de la educación física y la teońa sistemática de turismo (Cynarski \& Obodyński, 2004a).

Todas estas tareas de investigación son foco de interés para los estudiosos de otros centros polacos, como por ejemplo Artur Litwin, que participó junto con otros durante años en la investigación respecto a la jerarquía de valor (Cynarski \& Litwiniuk, 2008). La IPA también reune a reconocidos expertos extranjeros que participan seriamente en los trabajos de la Escuela de Rzeszów. Entre ellos están el portugués Abel A. Figueiredo ( 6 dan), cooperando en los estudios sobre institucionalización de las artes marciales (Figueiredo, 2009), el alemén Lothar Sieber ( 10 dan), co-autor de mudhos estudios de la IPA, o los autores A. Borbély (Hungría), C. Gutiérez (España), S.R. Hoogey K.R. Kemspedht (Alemania), R.. Maroteaux (Francia), o S. Shimizu (Japón) entre otros, autores de trabajos publicados en IRK-MC.

Como señáó Kazimierz Obodyński, “日 discumir de las tradiciones de extremo oriente, de diálogo cultura y de las distintas dimensiones humanísticas son examinadas en muchos aspectos por Cynarski y el círculo de científicos asociados con IRK-MC. Cynarski presenta una teoría humanística sistemática de las artes merciales diseñada a partir del ejemplo del budo japonés. 日 equipo compuesto por estudiosos de Rzeszów e intemacionales está desarrollando la teoría, construyendo una teoría genera sobre los caminos de las artes marciales y de los deportes de combate La base teórica se construye aquí sobre la antropología integral y la filosofía específica de las artes marciales, los logros de la sociología humenística y la sociología polaca de la cultura física, la pedagogía holística y la teoría humanística de la cultura física. La multi-disciplinariedad y los esfuerzos realizados, junto con el carácter sistemático de las investigaciones de estos expertos, son dignos de reconocimiento, y sus resultados, tanto en téminos ge nerales como particulares, merecen difundirse, considerando el hedho de que son poco conocidos fuera de Polonia La teoría humenística de las artes marciales y la antropología holística de las artes marciales, la cual conforma su base, presentan el canon de reflexión humanística del fenómeno mundial de las artes marciales y los deportes de combate Con gran satisfacción me gustańa añadir que estos conceptos han sido desarrollados por el profesor Cynarski en el centro académico de Rzeszów. Debido a ese hedho, se hizo posible la fundación de la primera publicación científica dedicada a la investigación humanística de las artes marciales - IRK-MC"' (Obodyński, 2008).

\section{Condusión}

Las principales direcciones de la investigación socio-cultural emprendidas en Polonia comprenden la historia y evolución de distintas artes marciales, análisis axiomáticos (incluyendo temes de valores y estilos de vida), recepción y entendimiento de las artes marciales, instituciones e institucionalización, participación social, diálogos culturales, espacio social, relaciones interpersonales en los grupos de practicantes, conexiones con el marketing y los negocios, así como el turismo de las artes marciales. Mudhos de 
estos temas han sido descritos y explicados con gran detalle en las investigaciones ya redizadas, mientras que otros, por el contrario, aún están en fases iniciales/preliminares, o simplemente se han señalado como temas de interés. Así, y a pesar del camino ya recomido, existen algunas tareas pendientes para la sociología de las artes marciales, en varios de los ámbitos definidos en este estudio, así como en otros ámbitos que aún requieren de una definición más exacta.

\section{BIBUOGRAFí}

Błach, W., Cynarski, W.J., Kowalski P., Migasiewic J., Tomeszewski M. \& Bładh Ł. (2006). History of participation and achievements of Polish representatives in world judo dhampionships (en polaco). Ido-Ruch dla Kultury / Movernent for Culture, 6, 63-6.

Borysiuk, Z (2005). Historical transformations of sabre as a fencing weapon (en polaco). Ido-Ruch dla Kultury / Movernent for Culture, 5, 9-15.

Castelnuovo, S. \& Guthrie, S.R. (1998). Ferinism and the Fermale Body: Liberating the Amazon Within. Boulder: L Rienner.

Chiba, N., Ebihara, O. \& Morino, S. (2001). Globalization, Naturalization and Identity. The Case of Borderless Eite Athletes in Japan. International Review for the Sociology of Sport, 36(2), 203-227

Cox, B. \& Thompson, S. (2000). Multiple Bodies: Sportswomen, Soccer and Sexuality. International Review for the Sociology of Sport, 35(1), 5-20.

Cynarski, W. J. \& Litwiniuk, A. (2008). Hierarchy of values in case of practitioners of far eastem mertial arts in Poland. International Joumal of Eastem Sports \& Physical Education, 6(1), 263-291

Cynarski, W. J. \& Obodyński, K. (2003a). Sociological reflection on contemporary phe nomenon of martial arts (en polaco). En Z Dziubiński [ed.], Spoleczny wymiar Sportu. Warszawa: SALOS RP, 188-195.

Cynarski, W. J. \& Obodyński, K. (2004a). "Tourist ways" of martial arts in Europesociological and cultural outline (en polaco). En M. Kazimierczak [ed.], Turystyka w humanistycznej perspektywie Poznań: AWF, 295-302.

Cynarski, W. J. \& Obodyński, K. (2004b). Modem sociology and sports philosophy in the face of socio-cultural issues of Far Eastem martial arts based on Polish and Germen papers from the years 1995-2001 En G. Anders, J. Mrazek, G. Norden, O. Weiss [eds.], European Integration and Sport. Münster. UT, 47-60.

Cynarski, W. J. \& Obodyński, K. (2005). Martial Arts in the process of institutional and ideological change on the example of Aikijutsu. International Journal of Eastern Sports \& Physical Education, 3(1), 118-129.

Cynarski, W. J. \& Obodyński, K. (2007). Sociology of Far Eastem mertial arts - problems and perspectives. Archives of Budo, 3(3), 49-53.

Cynarski, W. J. \& Obodyniski, K. [eds.] (2003b). Humanistic theory of martial arts and combat sportsconcepts and problems (en polaco). Rzeszów: University of Rzeszów.

Cynarski, W. J. (1997). Tradition of old Japanese aiki-jutsu and its evolution into currently practised forms (en polaco). Roczniki NaukoweAWF w Warszawie, 36, 109-132. 
Cynarski, W. J. (1999). Sociological and anthropological conditions of the Far Eastem fighting arts on the example of Japanese budō. Research Yearbook. Studies in the Theory of Physical Education and Sport, 6, 75-88.

Cynarski, W. J. (2000). Budo martial arts in the western culture (en polaco). Rzeszów: WSP.

Cynarski, W. J. (2003). Activities of Idokan Poland Association in the area of Podkanpa cie En M. Malikowski [ed.], Societies in Podkarpacie at the beginning of $2^{\text {st }}$ century. Sociological studies (en polaco). Rzeszów: Mana, 347-355.

Cynarski, W. J. (2004). Theory and practice of Far-Easter martial arts in European Perspective (en polaco). Rzeszów: University of Rzeszów.

Cynarski, W. J. (2005a). Architecture and anthropology of Far-Easter martial arts: dōjō ardhitecture spatial sociology outline (en polaco). Id $\bar{b}-R u c h$ dla Kultury / Movement for Culture, 5, 75-95.

Cynarski, W. J. (2005b). Sociological Aspects of Far Eastem Martial Arts. Reseerch Yearbook. Studies in Physical Education and Sport, 11, 103-108.

Cynarski, W. J. (2005c). Activities of Idokan Poland Association and Polish Union of Kobudō and Aikibudō between the year 1987 and 2000 (en polaco). Kultura Fizyczna. Prace Naukowe Akademii im J. Długosza w Częstochowie, 6, 8795.

Cynarski, W. J. (2006a). National sports traditions versus globalization. Sociological interpretation. Poland and its easter neighbours. Studia Wschodnioznawcze (en polaco), Rzeszów: University of Rzeszów, vol. 7, 114-122.

Cynarski, W. J. (2006b). The institutionalization of martial arts. European Joumal for Sport and Society, 3(1), 55-61

Cynarski, W. J. (2008a). Meetings, conflicts and dialogues. Analysis of the selected areas of physical culture and cultural tourism (en polaco). Rzeszów: University of Rzeszów.

Cynarski, W. J. (2008b). Una visión general de las Artes Marciales Polacas. Revista de Artes Marciales Asiaticas, 3(3), 8-25.

Cynarski, W. J. (2008c). Westemization of Far-Eastem martial arts and eastemization of the westem physical culture on the example of jūjutsu (en polaco). En W.J. Cynarski, A. Nowakowski, S. Zabomiak [eds.], Studia z historii i teorii kultury fizycznej. Księga jubileuszowa dedykowana Profesorowi Kazimierzowi Obodyńskiemu. Rzeszów: University of Rzeszów, 375-385.

Cynarski, W. J. [ed.] (2009). Martial Arts \& Combat Sports - Humanistic Outlook, Rzeszów: Rzeszów University Press.

Cynarski, W. J., Obodyński, K., Litwiniuk, A. \& Cynarska, E (2006). Aikjutsu in Europe and in Poland - present-day picture (1987-2004). Idō - Ruch dla Kultury / Movement for Culture, 6, 68-82.

Cynarski, W. J., Sieber, L \& Litwiniuk, A. (2005). Perception, understanding and adaptation of Asian martial arts in the West: a sociological analysis. Archives of Budo, 1, 13-18.

Cynarski, W. J., Sieber, L \& Litwiniuk, A. (2006). Asian martial arts in European and American reception (en polaco). Idō - Ruch dla Kultury / Movement for Culture (IRK-MC), 6, 252-260.

Czajkowski, Z (2007). Fencing in ancient Poland (en polaco). Idō - Ruch dla Kultury / Movement for Culture, 7, 55-64.

Donohue, J. J. (1994). Warriors Dreams. The Martial Arts and the American Imagination. Westport, Connecticut - London: Bergin \& Garvey. 
Donohue J. J. (1997). Ideological elasticity: Enduring form \& changing function in the Japanese martial tradition. Joumal of Asian Martial Arts, 6(2), 10-25.

Edhberg, H. (2004). Force Against Force Configurations of Martial Art in European and Indonesian Cultures. Id $\bar{b}$-Ruch dla Kultury / Movernent for Culture 4, 262285.

Figueiredo, A. A. (2009). The object of study in martial arts and combat sports research - contribution to a complex whole En W.J. Cynarski [ed.], Martial Arts \& Combat Sports - Humanistic Oudlook. Rzeszów: Rzeszów University Press, 20-34.

Förster, A. (1983). Neue Perspektiven für den Sport durch diePhilosophie und Praxis der fernöstlichen Kampfkünste En H. Lenk [Ed.], Aktuelle Probleme der Sportphilosophie Schomdorf: K. Hofmann, 271-240.

Grzegorz, R. \& Walendowicz, M. (2008). The History of Jutitsu in Poland 1904-1939. Poznań: IFMJJ \& AWF.

Hart, J., Faber, U. \& Bögle, R. (1989). Taekwon-Do imWesten. Interviews und Beiträge zumkulturellen Schlagabtausch. München: Mönchseulen-Verlag.

Hovden, J. (2000). Gender and Leadership Selection Processes in Norwegian Sporting Organisations. International Review for the Sociology of Sport, 35(1), 75-82.

Jaskólski, E (2000). Personality and body disposition development in the pedagogical process of using Budo rules. En R.M. Kalina, W. Jagielło [eds.], Educational and utilitarian aspects of combat sports (en polaco). Warszawa: AWF, 9-13.

Jaskólski, E, Migasiewicz, J. \& Kurzawski, K. (2008). Pedagogy of eastem martial artssuggestions for contemporary education (en polaco). En W.J. Cynarski, A. Nowakowski, S. Zabomiak [eds.], Studia z historii i teorii kultury fizycznej. Rzeszów: University of Rzeszów, 391-394.

Kalina, R. M. (2000). Theory of combat sports (en polaco). Warszava: COS.

Kosiewicz, J. (2005). Humanistic theory of mertial arts (en polaco). Ido-Ruch dla Kultury / Movement for Culture, 5, 279-227

Kosievicz, J. (2007). Philosophy of physical culture in Poland (en polaco). Ido-Ruch dla Kultury / Movement for Culture, 7, 11-37.

Litwiniuk, A. \& Cynarski, W. J. (2001). Fair play idea in the conscience of people practising selected sports and martial arts (en polaco). Idō-Ruch dla Kultury / Move ment for Culture, 2, 144-149.

Maeda, H. (2000). Prosperous Middleaged Women's Sports in Japan through Some Gender Issues. International Review for the Sociology of Sport, 35(1), 487494.

Maroteaux, R. J. \& Cynarski, W. J. (2002-2003). About the philosophy of Japanese martial arts (en polaco). Id $\bar{o}-R u c h$ dla Kultury / Movement for Culture, 3, 48-55.

McDonald, M.G. (2000). The Marketing of the Women's National Basketball Association and the Making of Postferinism International Review for the Sociology of Sport, 35(1), 35-47.

Mennesson, C. (2000). 'Hard' Women and 'Soft' Women: The Social Construction of Identities among Femele Boxers. International Review for the Sociology of Sport, 35(1), 27-33.

Mrówka, M. (2008). Historical cultural conditions of far eastem martial arts in the context of strategic management in modem industry of Japan and Korea (en polaco). Id $\bar{o}-$ Ruch dla Kultury / Movement for Culture, 8, 45-55. 
Obodyński, K. \& Cynarski, W. J. (2003). Asian martial arts in the process of global cultural exchanges. En J. Kosiewicz, K. Obodyński [eds.], Sport in the Mirror of the Values. Rzeszów: PTNKF, 119-130.

Obodyński, K. \& Cynarski, W. J. (2005). Social and Philosophical Determinants of the Lifestyle of a Contemporary Student of Martial Arts. En B. Hodan̆ [ed.], Tĕlesná vychova, sport a rekreace $v$ procesu současné globalizace Olomouc: Univerzita Paladkeho (República Checa), 271-281.

Obodyński, K. (2008). Anthropology of martial arts as a scientific perspective for re search (en polaco). Przeglad Naukowy Kultury Fizycznej UR, 11(1), 11-20.

Orlando, B. (1997). Martial arts America: a Westem approach to Eastem arts. Berkeley, Calif.: Frog, North Atlantic Books.

Pater, R. (1990/1999). Leading from within: martial arts skills for dynamic business and management Rochester, Vt.: Park Street Press.

Pantuddi, A. (2004). Review of "Scientific Year's Issue Idō - Cultural Movement" ed. Wojciech Cynarski, Rzeszów 2002, vol. 3, Biblioteka Lykeion. Id̄̄-Ruch dla Kultury / Movement for Culture, 4, 12-15.

Pantudki, A. (2007). Asian body cult in the Polish way - imperialism or diffusion (en polaco). En A. Pantudk, Person in body pedagogy. Olimpic peacelaw. GdańskOlsztyn, 188-202.

Sieber, L \& Cynarski, W. J. (2004). The masters' masters from WJJC federation - new facts (en polaco). Idō-Ruch dla Kultury / Movement for Culture, 4, 293-297.

Ślęzak, M. (2006). Using narrative interview on the basis of physical culture sociology with particular emphasis on Far-Eastem martial arts (en polaco). Id $\bar{o}-$ Ruch dla Kultury / Movement for Culture, 6, 34-41

Socha, N. (2001). Damska pięść. Wprost, 20, 72.

Speer, S.A. (2001). Review: S. Castelnuovo, S. R. Guthrie Ferinism and the Femble Body: Liberating the Amazon Within. International Review for the Sociology of Sport, 36(1), 89-91

Sterkowicz, S. (2001). Systematic and cultural analysis of martial arts in view of W.J. Cynarski (en polaco). Przeglad Naukowy IWFZ UR 4, 481-483.

Sterkowic, S. (2003). Special skills and a social position in hapkido group. En W.J. Cynarski, K. Obodyński [eds.] Humanistic theory of martial arts and combat sports-concepts and problems (en polaco). Rzeszów: University of Rzeszów, 79-83.

Szyszko-Bohusz, A. (2003). Theory of genetic immortality in relation to holistic pedagogy and Far-Eastem martial arts (en polaco). En W.J. Cynarski, K. Obodyński [eds.], Humanistic theory of martial arts and combat sports-concepts and problemb. Rzeszów: University of Rzeszów, 17-23.

Szyszko-Bohusz, A. (2008a). A learner of Far-Eastem martial arts faced with extreme situations (en polaco). En W.J. Cynarski, A. Nowakowski, S. Zabomiak [eds.], Studia z histoni i teoni kultury fizycznej. Rzeszów: University of Rzeszów, 386-390.

Szyszko-Bohusz, A. (2008b, noviembre). Entrevista personal con el autor.

Tokarski, S. \& Sikorski, W. (2009). Martial arts at the cross-roads - Asiatic westemiza tion or Westem eastemization. En W.J. Cynarski [ed.], Martial Arts \& Combat Sports - Humanistic Outlook. Rzeszów: University of Rzeszów, 77-82.

Tokarski, S. (1976). The second Renaissance Eiade's theory of cross-cultural dialogues (en polaco). Kultura i Społeczeístwo, 1, 149-163. 
Tokarski, S. (1989). Martial arts. Activeforms of expression in the philosophy of theEast (en polaco), Szczedin: Glob.

Tokarski, S. (2003). Paradox of martial arts of Central Asia - Westem pattems of expansion and Eastem Values. En J. Kosievicz, K. Obodyński [eds.], Sport in the Mirror of the Values. Rzeszów: PTNKF, 112-118.

Villamón, M., Brown, D., Espartero, J. \& Gutiérez, C. (2004). Reflexive Modemization and the Disembedding of Jū $\overline{0}$ from 1946 to the 2000 Sydney Olympics. International Review for the Sociology of Sport, 39(2), 139-156.

Zablodk, W. (2000). The Polish martial arts. Two-handed sword and hussar sabre (en polaco). Idō-Ruch dla Kultury / Movement for Culture, 1, 114-115. 